\title{
A Precise Definition of an Inference (by the Example of Natural Deduction Systems for Logics $\left.I_{\langle\alpha, \beta\rangle}\right)^{1}$
}

\section{Shangin Vasilyi Olegovich}

Department of Logic, Faculty of Philosophy, Lomonosov Moscow State University. Lomonosovsky prospect, 27-4, GSP-1, Moscow, 119991, Russian Federation.

E-mail: shangin@philos.msu.ru

In the paper, we reconsider a precise definition of a natural deduction inference given by V. Smirnov. In refining the definition, we argue that all the other indirect rules of inference in a system can be considered as special cases of the implication introduction rule in a sense that if one of those rules can be applied then the implication introduction rule can be applied, either, but not vice versa. As an example, we use logics $I_{\langle\alpha, \beta\rangle}, \alpha, \beta \in\{0,1,2,3, \ldots \omega\}$, such that $I_{\langle 0,0\rangle}$ is propositional classical logic, presented by V. Popov. He uses these logics, in particular, a Hilbertstyle calculus $H I_{\langle\alpha, \beta\rangle}, \alpha, \beta \in\{0,1,2,3, \ldots \omega\}$, for each logic in question, in order to construct examples of effects of Glivenko theorem's generalization. Here we, first, propose a subordinated natural deduction system $N I_{\langle\alpha, \beta\rangle}, \alpha, \beta \in\{0,1,2,3, \ldots \omega\}$, for each logic in question, with a precise definition of a $N I_{\langle\alpha, \beta\rangle}$-inference. Moreover, we, comparatively, analyze precise and traditional definitions. Second, we prove that, for each $\alpha, \beta \in\{0,1,2,3, \ldots \omega\}$, a Hilbert-style calculus $H I_{\langle\alpha, \beta\rangle}$ and a natural deduction system $N I_{\langle\alpha, \beta\rangle}$ are equipollent, that is, a formula $A$ is provable in $H I_{\langle\alpha, \beta\rangle}$ iff $A$ is provable in $N I_{\langle\alpha, \beta\rangle}$.

Keywords: precise definition of inference, indirect rule, implication introduction rule, natural deduction, quasi-elemental formula, subordinated sequence

\section{Introduction}

In [10], V. Popov presents logics $I_{\langle\alpha, \beta\rangle}$ and Hilbert-style calculi $H I_{\langle\alpha, \beta\rangle}, \alpha, \beta \in 0,1,2,3, \ldots \omega$, for these logics, such that $I_{\langle 0,0\rangle}$ is propositional classical logic. He uses them in order to construct examples

\footnotetext{
${ }^{1}$ The author is supported by Russian Foundation for Humanities, grant 16-0300749 "Logical-epistemic problems of knowledge representation".
} 
of effects of a generalization of Glivenko theorem. So, the purpose of the present paper is to present, within the framework of [8, 9], a subordinated natural deduction (abbreviated passim as 'ND') calculus $N I_{\langle\alpha, \beta\rangle}$, for each logic in question, with the precise definition of an $N I_{\langle\alpha, \beta\rangle}$-inference, following the works of V. Smirnov $[12,14]$. We, also, show the equipollentness between a Hilbert-style calculus $H I_{\langle\alpha, \beta\rangle}$ and a ND system $N I_{\langle\alpha, \beta\rangle}$, for each $\alpha, \beta \in\{0,1,2,3, \ldots \omega\}$, that is, a formula A is provable in $H I_{\langle\alpha, \beta\rangle}$ iff $\mathrm{A}$ is provable in $N I_{\langle\alpha, \beta\rangle}$.

Following [10], we fix a standard propositional language $L$ over an alphabet $\left\{p, p_{1}, p_{2}, \ldots,(), \&,, \vee, \supset, \neg\right\}$. A notion of a formula of language $L$ is defined as usual. (Passim by 'a formula' we mean 'a formula of language $L^{\prime}$.) A formula is said to be quasi-elemental iff no logical connective \&, $\vee, \supset$ occurs in it ([10]). A length of a formula A is said to be the number of all occurrences of the logical connectives in $L$ in $\mathrm{A}$. Letters A, B, C, D, E with lower indexes run over arbitrary formulae. Letters $\Gamma, \Delta$ with upper and lower indexes run over arbitrary finite sets of formulae. Letters $\alpha$ and $\beta$ run over $\{0,1,2,3, \ldots \omega\}$ passim.

In [10], V. Popov presents a Hilbert-style calculus $H I_{\langle\alpha, \beta\rangle}$. The language of the calculus is the language $L$ mentioned above. We follow (and, for more details, refer the reader to) [10] in describing a Hilbertstyle calculus $H I_{\langle\alpha, \beta\rangle}$. A formula is an axiom of $H I_{\langle\alpha, \beta\rangle}$ iff it is one of the following forms: (I) $(A \supset B) \supset((B \supset C) \supset(A \supset C))$, (II) $A \supset(A \vee B)$, (III) $B \supset(A \vee B),(\mathrm{IV})(A \supset C) \supset((B \supset C) \supset((A \vee B) \supset C))$, $(\mathrm{V})(A \& B) \supset A,(\mathrm{VI})(A \& B) \supset B,(\mathrm{VII})(C \supset A) \supset((C \supset B) \supset(C \supset$ $(A \& B))),(\mathrm{VIII})(A \supset(B \supset C)) \supset((A \& B) \supset C),(\mathrm{IX})((A \& B) \supset C) \supset$ $(A \supset(B \supset C)),(\mathrm{X})(((A \supset B) \supset A) \supset A),(\mathrm{XI}, \alpha) \neg D \supset(D \supset A)$, where $D$ is a formula which is not a quasi-elemental formula of a length less than $\alpha$, (XII, $\beta)(E \supset \neg(A \supset A)) \supset \neg E$, where $E$ is a formula which is not a quasi-elemental formula of a length less than $\beta$. Modus ponens is the only inference rule of the calculus.

Definitions of an inference in $H I_{\langle\alpha, \beta\rangle}$ (abbreviated as $H I_{\langle\alpha, \beta\rangle^{-}}$ inference) and a proof in $H I_{\langle\alpha, \beta\rangle}$ are given in the standard way for a Hilbert-style calculus. Notions of the length of an inference and the length of a proof as well as the notion of a theorem are defined as usual. 
In [10], the following fact is particularly highlighted: $I_{\langle 0,0\rangle}$ is propositional classical logic, where $I_{\langle 0,0\rangle}$ is the set of formulae provable in $H I_{\langle 0,0\rangle}$. This fact implies both schemata $\mathrm{A} \supset(\mathrm{B} \supset \mathrm{A})$ and $(A \supset(B \supset$ $C)) \supset((A \supset B) \supset(A \supset C))$ are theorems of $H I_{\langle 0,0\rangle}$ and, therefore, of each Hilbert-style calculus $H I_{\langle\alpha, \beta\rangle}, \alpha, \beta \in\{0,1,2,3, \ldots \omega\}$. So, we, nonconstructively, point out the standard deduction theorem holds for each calculus in question.

The paper is organized as follows. Section 1 presents a ND system $N I_{\langle\alpha, \beta\rangle}$ with both precise and traditional definitions of an $N I_{\langle\alpha, \beta\rangle^{-}}$ inference. In Section 2, the Hilbert-style calculus $N I_{\langle\alpha, \beta\rangle}$ and the ND system $N I_{\langle\alpha, \beta\rangle}$ are shown to be equipollent. The final section concludes the work and outlines the future research.

\section{ND systems $N I_{\langle\alpha, \beta\rangle}$}

Let us set up a subordinated ND system $N I_{\langle\alpha, \beta\rangle}$ and give a precise definition of a $N I_{\langle\alpha, \beta\rangle}$-inference. The language of the system is, again, the language $L$ mentioned above. There are two kinds of rules in the system. Here is the list of the rules of the first kind (sometimes called direct). The rules of the second kind (sometimes called indirect) are defined with the precise definition of an inference below.

$$
\begin{array}{lll}
\frac{A \& B}{A} \&_{e l 1} & \frac{A \& B}{B} \&_{e l 2} & \frac{A, B}{A \& B} \&_{i n} \\
\frac{A}{A \vee B} \vee_{i n 1} & \frac{B}{A \vee B} \vee_{i n 1} & \frac{A \supset B, A}{B} \supset_{e l}
\end{array}
$$

$\frac{D, \neg D}{A} \neg_{i n 1(\alpha)}$, where $D$ is a formula which is not a quasi-elemental formula of a length less than $\alpha$.

The necessity of a precise definition of $N I_{\langle\alpha, \beta\rangle}$-inference is illustrated with V. Smirnov's thesis: “... By natural deduction systems we shall refer to logistic systems with a special notion of an inference. In these systems, an inference is more complex object than just a sequence of formulae or a tree-like of formulae. Due to this property of natural deduction systems, a definite object entitled a formal inference corresponds to both direct 
and indirect ways of argument" [12, p. 96, both the translation and the italics are ours].

In defining both a $N I_{\langle\alpha, \beta\rangle}$-inference и its length, we, with modifications, follow V. Smirnov [12, p. 116-118], [14, p. 245]. Letters $\eta$ и $\iota$ with indexes denote $N I_{\langle\alpha, \beta\rangle}$-inferences, a letter $\gamma$ with indexes denotes parts of $N I_{\langle\alpha, \beta\rangle}$-inferences, and a letter h denotes the length of an inference. ${ }^{2}$

An precise definition of $N I_{\langle\alpha, \beta\rangle}$-inference and a definition a height of $N I_{\langle\alpha, \beta\rangle}$-inference

1. $\mathrm{A}$ is an inference $\iota$ of $\mathrm{A}$ from a set of premises $\{A\}$, and $h(\iota)=1$.

2. If $\eta$ is an inference from $\Gamma$ and $\mathrm{A}$ is a formula, then ${ }_{A}^{\eta}$ is an inference $\iota$ of $\mathrm{A}$ from $\{A\} \cup \Gamma$, and $h(\iota)=h(\eta)+1$.

3. If $\eta$ is an inference from $\Gamma, \eta$ contains $A_{1}, \ldots, A_{k}(k=1,2)$ and $B$ is inferred from $A_{1}, \ldots, A_{k}$ via one of the rules $\&_{e l 1}, \&_{e l 2}, \&_{i n}$, $\vee_{i n 1}, \vee_{i n 2}, \supset_{e l}$ and $\neg_{i n 1(\alpha)}$, then $\underset{B}{\eta}$ is an inference $\iota$ of $\mathrm{B}$ from $\Gamma$, and $h(\iota)=h(\eta)+1$.

4. If $\eta$ is an inference of $B$ from $\{A\} \cup \Gamma$ and $\eta$ is $\underset{\gamma_{1}}{\gamma}$, where $\gamma_{1}$ is a part of $\eta$, starting from the last premise A in $\eta$ until B itself, ${ }^{3}$ then $\underset{A \supset B}{\mid \gamma_{1}}$ is an inference $\iota$ of $A \supset B$ from $\Gamma$, and $h(\iota)=h(\eta)+1$.

5. If $\eta$ is an inference of $B$ from $\{A\} \cup \Gamma$ and $\eta$ is $\underset{\gamma}{\gamma}$, where $\mathrm{B}$ is $\mathrm{C}, \mathrm{A}$ is $\mathrm{C} \supset D, \gamma_{1}$ is a part of $\eta$, starting from the last premise $C \supset D$ in $\eta$ until $C$ itself, then $\underset{C}{\mid \gamma_{1}}$ is an inference $\iota$ of $C$ from $\Gamma$, and $h(\iota)=h(\eta)+1$.

6. If $\eta$ is an inference of $B$ from $\{A\} \cup \Gamma$ and $\eta$ is $\underset{\gamma}{\gamma}$, where $\mathrm{B}$ is $\neg(A \supset A), \mathrm{A}$ is $E$, where $E$ is a formula which is not a quasielemental formula of a length less than $\beta$ and $\gamma_{1}$ is a part of $\eta$,

\footnotetext{
${ }^{2}$ In every case, the precise definition specifies which part of a $N I_{\langle\alpha, \beta\rangle}$-inference is under consideration. The reason we introduce a special letter to run over parts of inference is that, in general, as we will see, a part of an inference is not an inference.

${ }^{3}$ It is the last occurrence of $B$ in $\eta$ that is under consideration. In what follows, we will omit this specification everywhere, except clause 7 .
} 
starting from the last premise $\mathrm{E}$ in $\eta$ until $\neg(A \supset A)$ itself, then $\underset{\neg E}{\mid \gamma_{1}}$ is an inference $\iota$ of $\neg E$ from $\Gamma$, and $h(\iota)=h(\eta)+1$.

7. If $\eta$ is an inference of $B$ from $\left\{D_{1}\right\} \cup\left\{D_{2}\right\} \cup \Gamma$ and $\eta$ is $\underset{\gamma_{2}}{\gamma}$, where B is $C$ and $\gamma$ contains $D_{1} \vee D_{2}, \gamma_{1}$ is a part of $\eta$, starting from the last premise $D_{1}$ in $\eta$ until $C, \gamma_{2}$ is a part of $\eta$, is a part of $\eta$, starting from the last premise $D_{2}$ in $\eta$ until $C$ itself, then $\underset{C}{\frac{\gamma}{\gamma_{2}}} \underset{C}{\gamma}$ is an inference $\iota$ of $C$ from $\Gamma$, and $h(\iota)=h(\eta)+1 .^{4}$

The core of modifications is as follows. An essential modification deals with V. Smirnov's suggestion that any discarded part of a $N I_{\langle\alpha, \beta\rangle^{-}}$ inference is a $N I_{\langle\alpha, \beta\rangle}$-inference. (A discarded part of an inference is marked with a horizontal line from the left.) This is not the case if a part of a $N I_{\langle\alpha, \beta\rangle}$-inference contains a formula that is not a premise and is inferred from the formulae which this part of a $N I_{\langle\alpha, \beta\rangle}$-inference does not contain. For example, in the clause 4 , a part $\gamma_{1}$ may contain a formula that is inferred from some formula contained in a part $\gamma$ (and $\gamma$ may be an inference, itself). So, $\gamma_{1}$ is not an inference while $\gamma_{1}^{\gamma}$ is. Sometimes, V. Smirnov applies a notion of an auxiliary inference (or a subderivation) to such sequences of formulae as $\gamma_{1}$. The name of this notion obviously reflects the idea that such an inference plays a secondary role, and can be considered only with respect to the 'key' inference. However, we can't find it satisfactory that an auxiliary inference is shown not to be a kind of an inference. At last, minor modifications deal with evaluating a height of $N I_{\langle\alpha, \beta\rangle}$-inference in $\supset_{e l}$ and $\vee_{e l}$ rules as well as with evaluating the height of a $N I_{\langle\alpha, \beta\rangle}$-inference that now cannot be equal to 0 .

Clause $4(5,6$, and 7 , respectfully) of the above definition is a formulation of an indirect rule of $\supset_{i n}\left(\supset_{P}, \neg_{i n 2(\beta)}\right.$, and $\vee_{e l}$, respectfully). We pay attention (and exemplify it below) to the fact that clauses 5-7 are special cases of clause 4 . (In case of clause 7 , the situation is a little bit

\footnotetext{
${ }^{4}$ Clause 7 may have alternative formulations: 1. $\gamma_{3}$ occurs between $\gamma_{1}$ and $\gamma_{2} ; 2$. $\gamma_{1}$ reorders with $\gamma_{2} ; 3 . D_{1} \vee D_{2}$ occurs below a part $\gamma_{1}$ etc. This analysis goes beyond the scope of the paper.
} 
more complex than in the other cases because it allows simultaneously discarding two parts of an inference, not one part. It is the reason why we choose clause 7 in the example below.) By the fact that a rule, say, $\supset_{P}$, is a special case of a rule $\supset_{i n}$ we mean that if one can apply $\supset_{p}$ in the inference then one can apply $\supset_{i n}$, either, but not vice versa. To be sure, we don't mean $\supset_{p}$ is derivable via $\supset_{i n}$.

There different notation formats for a subordinated inference in ND systems [12, p. 119-126]. We will use so called Jaskowski-Quine notation in $[2] .^{5}$

Let us consider the following sequence of formulae:

1. $A \supset C$ - premise.

2. $B \supset C$ - premise.

3. $A \vee B-$ premise.

4. $A$ - premise.

5. $C-\supset_{e l}: 1,4$

6. $B$ - premise.

7. $C-\supset_{e l}: 2,6$

In accordance to clause 4, we have an inference of $C$ from premises $A \supset C, B \supset C, A \vee B, A$, and $B$. Thus, we are legitimate to proceed with an inference of $B \supset C$ from premises $A \supset C, B \supset C, A \vee B$, and $A$ :

1. $A \supset C$-premise.

2. $B \supset C-$ premise.

3. $A \vee B-$ premise.

4. $A$ - premise.

5. $C-\supset_{e l}: 1,4$

|6. $B$ - premise.

17. $C-\supset_{e l}: 2,6$

8. $B \supset C-\supset_{i n}: 7$

\footnotetext{
${ }^{5}$ In the literature, a subordinated inference is sometimes called a linear-type ND or a Fitch-style ND [11]. A subordinated inference differs from a tree-like inference presented by G. Gentzen [5], where, roughly, no formula is used more than once in the inference as a premise.
} 
On the other hand, in accordance to clause 7 , an inference of $C$ from premises $A \supset C, B \supset C, A \vee B, A$, and $B$ contains a part, starting from the last premise $A$ until $C$ (steps $4-5$ ), and a part, starting from the last premise $B$ until $C$ (steps 6-7), as well as it contains $A \vee B$ which contains in no parts mentioned above. Thus, we are legitimate to proceed with an inference of $C$ from premises $A \supset C, B \supset C$, and $A \vee B$ :

1. $A \supset C$ - premise.

2. $B \supset C$ - premise.

3. $A \vee B$ - premise.

14. $A$ - premise.

15. $C-\supset_{e l}: 1,4$

|6. $B$ - premise.

17. $C-\supset_{e l}: 2,6$

8. $C-\vee_{i n}: 7$

As a result, we see the complexity of a notion of an inference in ND systems leads to the fact that a sequence of formulae turns out to be different inferences of the same formula from different set of premises. Discussing this fact (which is impossible for the other conventional proof systems like Hilbert-style calculus, sequent-style calculus and tree-like ND system) and its consequences is not a topic of the paper. We are fully aware, however, that the fact that a precise definition of an inference leads to some ambivalence seems to be absurd. But we strongly believe that the reason of this fact is caused by the nature of indirect argument, itself, which have been being under suspicion in the development of logic. ${ }^{6}$

On the other hand, the difference between direct and indirect rules has become more evident. A direct rule is applicable provided an inference contains formula (formulae) which is (are) above the line in a formulation of this some rule. One can apply a direct rule to any formula; it is not necessary for the formula to be the last one in this inference. For example, in applying $\&_{e l 1}$ or some other direct rule, A\&B (the one

\footnotetext{
${ }^{6}$ It is well-known that intuitionists have been criticizing the general version of reductio ad absurdum, a type of indirect argument.
} 
that is above the line) is not necessary the last formula of the inference, i.e., it is not necessary that this inference is an inference of $A \& B$ from (possibly, empty) $\Gamma$.

The situation is not the same in case of indirect rules. An indirect rule is applicable, too, provided there is an inference of the formula which is above the line in the formulation of this rule. The crucial difference is that it applies to the last formula in an inference only. (Note, at any moment, there is only one formula that is the last one in an inference.) For example, in applying $\supset_{i n}$, a formula B (the one that is above the line) is, necessarily, the last one in an inference, i.e., it is a must that there is an inference of $\mathrm{B}$ from a non-empty set of premises $\Gamma$, where $A$ is a member of $\Gamma$. In the example above, we see that one has different options in applying indirect rules in the same way one has different options in applying direct rules. However, it is impossible for a sequence of formulae to be an inference of two formulae: such a possibility is allowed by so called traditional formulation of some indirect rules (in the next subsection it holds for the traditional formulation of $\vee_{e l}$ ). Sometimes negation introduction rule is formulated, roughly, as follows: if there is an inference of both formulae $A$ and $\neg A$ from the last premise $C$ then there is an inference of a formula $\neg C$ [1, p. 140], or 'to be applied, an indirect rule $\neg$ in requires two auxiliary inferences $\Gamma, A \vdash B$ and $\Gamma, A \vdash \neg B^{\prime}[13$, p. $66]$. In general, one can't determine another inference (so called 'auxiliary inference') 'inside' a given inference. Let us, again, say that discussing it goes beyond the scope of this paper.

In the end of this section, let us present so called the traditional formulations of both indirect rules and of an inference. ${ }^{7}$ In the rules below, a formula $A$ ( $A \supset B$ or $E$ ) is the last premise. In $\neg_{i n 2(\beta)}$, a formula $E$ is, additionally, a formula which is not a quasi-elemental formula of a length less than $\beta$. In this subsection, by 'inference' we mean ' $N I_{\langle\alpha, \beta\rangle}$ ' inference'.

\footnotetext{
${ }^{7}$ For the sake of simplicity and without loss of generality, we don't present a traditional formulation of $\vee_{e l}$ and refer the reader to, for example, [6]. Note, sometimes, the traditional formulation of the indirect rules includes the derivability symbol ' $\vdash$ ' [1].
} 


$$
\frac{[A] B}{A \supset B} \supset_{i n} \quad \frac{[A \supset B] A}{A} \supset_{p} \quad \frac{[E] \neg(B \supset B)}{\neg E} \neg_{i n 2(\beta)}
$$

An inference is said to be a non-empty finite linearly ordered sequence of formulae $C_{1}, C_{2}, \ldots, C_{k}$, satisfying the following conditions: ${ }^{8}$

- Each $C_{i}$ is either a premise or is inferred from the previous formulae via a rule;

- In applying $\supset_{i n}$, each formula, starting from the last premise $A$ until $A \supset B$, the result of this application, exclusively, is discarded from an inference;

- In applying $\supset_{P}$, each formula, starting from the last premise $A \supset B$ until $A$, the result of this application, exclusively, is discarded from an inference;

- In applying $\neg_{i n 2(\beta)}$, each formula, starting from the last premise $E$ until $\neg E$, the result of this application, exclusively, is discarded from an inference.

Given an inference $C_{1}, C_{2}, \ldots, C_{k}$ with $A_{1}, A_{2}, \ldots, A_{n}$ being nondiscarded premises and with the last formula $C_{k}$ being graphically identical to $B$, we say this is an inference of $B$ from premises $A_{1}, A_{2}, \ldots, A_{n}$. If a set of formulae $\Gamma$ contains $A_{1}, A_{2}, \ldots, A_{n}$ and there is an inference of $B$ from premises $A_{1}, A_{2}, \ldots, A_{n}$ then we say there is an inference of $B$ from a set of formulae $\Gamma$ [2, p. 129-130].

\section{Metatheory of a ND system}

We proof the following

Theorem 1. $\Gamma \vdash_{H I\langle\alpha, \beta\rangle} A \Leftrightarrow \Gamma \vdash_{N I\langle\alpha, \beta\rangle} A$, for each $\alpha, \beta \in$ $\{0,1,2,3, \ldots \omega\}$.

\footnotetext{
${ }^{8}$ Here is (of course, incomplete) a list of (text)books reproducing the traditional formulation one way or another: [1]-[4], [6], [15], [16]. On the other hand, we are fully aware that textbooks' authors are, mostly, driven by pedagogy trying to 'not go deep into theoretical subtleties of all kinds' and following the principle 'to tell the truth and only the truth, but not all the truth' [2, p. 11, 12].
} 
Proof $\Rightarrow$. Proof is by the method of complete induction on a height s of an arbitrary $H I_{\langle\alpha, \beta\rangle}$-inference of $A$ from $\Gamma{ }^{9}$

The scheme of complete induction is as follows: $(P(1) \& \forall x(\forall y((y<$ $x) \supset P(y)) \supset P(x))) \supset \forall x P(x)$.

Let $P(s)$ denote a sentence "if there is a $H I_{\langle\alpha, \beta\rangle}$-inference of a height $s$ of $A$ from $\Gamma$ then there is a $N I_{\langle\alpha, \beta\rangle}$-inference of $A$ from $\Gamma$ ".

Then the scheme looks as follows: ((if there is a $H I_{\langle\alpha, \beta\rangle}$-inference of a height 1 of $A$ from $\Gamma$ then there is a $N I_{\langle\alpha, \beta\rangle}$-inference of $A$ from $\Gamma$ ) $\& \forall s\left(\forall t\left((t<s) \supset\right.\right.$ (if there is a $H I_{\langle\alpha, \beta\rangle}$-inference of a height $\mathrm{t}$ of $A$ from $\Gamma$ then there is a $N I_{\langle\alpha, \beta\rangle}$-inference of $A$ from $\left.\Gamma\right)$ ) $\supset$ (if there is a $H I_{\langle\alpha, \beta\rangle^{-}}$ inference of a height $s$ of $A$ from $\Gamma$ then there is a $N I_{\langle\alpha, \beta\rangle}$-inference of $A$ from $\Gamma))) \supset \forall s\left(\right.$ if there is a $H I_{\langle\alpha, \beta\rangle}$-inference of a height $s$ of $A$ from $\Gamma$ then there is a $N I_{\langle\alpha, \beta\rangle}$-inference of $A$ from $\Gamma$ ).

The base case is trivial according to the definitions of inferences in both $H I_{\langle\alpha, \beta\rangle}$ and $N I_{\langle\alpha, \beta\rangle}$.

We prove the inductive step: $\forall s\left(\forall t\left((t<s) \supset\right.\right.$ (if there is a $H I_{\langle\alpha, \beta\rangle^{-}}$ inference of a height $t$ of $A$ from $\Gamma$ then there is a $N I_{\langle\alpha, \beta\rangle}$-inference of $A$ from $\Gamma)) \supset$ (if there is a $H I_{\langle\alpha, \beta\rangle}$-inference of a height $s$ of $A$ from $\Gamma$ then there is a $N I_{\langle\alpha, \beta\rangle}$-inference of $A$ from $\left.\Gamma\right)$ ).

For modus ponens is an inference rule in both $H I_{\langle\alpha, \beta\rangle}$ and $N I_{\langle\alpha, \beta\rangle}$, it is enough to show that every $H I_{\langle\alpha, \beta\rangle}$-axiom is provable in $N I_{\langle\alpha, \beta\rangle}$. We confine ourselves to proving two specific $H I_{\langle\alpha, \beta\rangle}$-axioms: axiom $(\mathrm{XI}, \alpha) \neg D \supset(D \supset A)$, where $D$ is a formula which is not a quasielemental formula of a length less than $\alpha$, and axiom (XII, $\beta)(E \supset$ $\neg(A \supset A)) \supset \neg E$, where $E$ is a formula which is not a quasi-elemental formula of a length less than $\beta$.

$$
\begin{aligned}
& \vdash_{N I_{\langle\alpha, \beta\rangle}} \neg D \supset(D \supset A) \\
& \mid 1 . \neg D-\text { premise } \\
& \| 2 . D-\text { premise } \\
& \| 3 . A-{ }_{i n 1}(\alpha): 1,2 \\
& \mid 4 . D \supset A-\supset \supset_{i n}: 3 \\
& \text { 5. } \neg D \supset(D \supset A)-\supset_{i n}: 4
\end{aligned}
$$

\footnotetext{
${ }^{9}$ We recall the standard definition of a length of an inference in a Hilbert-style calculus.
} 
$\vdash_{N I_{\langle\alpha, \beta\rangle}}(E \supset \neg(A \supset A)) \supset \neg E$

|1. $E \supset \neg(A \supset A)-$ premise

||2. $E-$ premise

$\| 3 . \neg(A \supset A)-\supset_{e l}: 1,2$

|4. $-_{i n 2(\beta)}: 3$

5. $(E \supset \neg(A \supset A)) \supset \neg E-\supset_{i n}: 4$

Proof $\Leftarrow$. Proof is by the method of complete induction on a height $n$ of an arbitrary $N I_{\langle\alpha, \beta\rangle}$-inference of $A$ from $\Gamma$.

The scheme of complete induction is as follows: $(Q(1) \& \forall x(\forall y((y<$ $x) \supset Q(y)) \supset Q(x))) \supset \forall x Q(x)$.

Let $Q(n)$ denote a sentence "if there is a $N I_{\langle\alpha, \beta\rangle}$-inference of a height $n$ of $A$ from $\Gamma$ then there is a $H I_{\langle\alpha, \beta\rangle}$-inference of $A$ from $\Gamma "$ ".

Then the scheme looks as follows: ((if there is a $N I_{\langle\alpha, \beta\rangle}$-inference of a height 1 of $A$ from $\Gamma$ then there is a $H I_{\langle\alpha, \beta\rangle}$-inference of $A$ from $\Gamma$ ) $\& \forall n\left(\forall q\left((q<n) \supset\right.\right.$ (if there is a $N I_{\langle\alpha, \beta\rangle}$-inference of a height $q$ of $A$ from $\Gamma$ then there is a $H I_{\langle\alpha, \beta\rangle}$-inference of $A$ from $\left.\Gamma\right)$ ) $\supset$ (if there is a $N I_{\langle\alpha, \beta\rangle^{-}}$ inference of a height $n$ of $A$ from $\Gamma$ then there is a $H I_{\langle\alpha, \beta\rangle}$-inference of $A$ from $\Gamma))) \supset \forall n\left(\right.$ ifthereisaN $I_{\langle\alpha, \beta\rangle}$-inference of a height $n$ of $A$ from $\Gamma$ then there is a $H I_{\langle\alpha, \beta\rangle}$-inference of $A$ from $\left.\Gamma\right)$.

The base case: $\mathrm{h}(\eta)=1$. According to clause 1 of the definition of a $N I_{\langle\alpha, \beta\rangle}$-inference, a $N I_{\langle\alpha, \beta\rangle}$-inference $\eta$ of a height 1 of A from a set of premises $\Gamma$ looks as follows: $A$ is an inference from $\{A\}$ :

1. A-premise.

This inference corresponds to the following $H I_{\langle\alpha, \beta\rangle}$-inference of $A$ from a set of premises $\{A\}$ :

1. A-premise.

We prove the inductive step: $\forall n\left(\forall q\left((q<n) \supset\right.\right.$ (if there is a $N I_{\langle\alpha, \beta\rangle^{-}}$ inference of a height $q$ of $A$ from $\Gamma$ then there is a $H I_{\langle\alpha, \beta\rangle}$-inference of $A$ from $\Gamma)$ ) $\supset$ (if there is a $N I_{\langle\alpha, \beta\rangle}$-inference of a height $n$ of $A$ from $\Gamma$ then there is a $H I_{\langle\alpha, \beta\rangle}$-inference of $A$ from $\left.\Gamma\right)$ ). According to clauses $2-7$ of the definition of a $N I_{\langle\alpha, \beta\rangle}$-inference, a $N I_{\langle\alpha, \beta\rangle}$-inference $\eta$ of a height $n$ of A from a set of premises $\Gamma$ looks as one of the six following cases: 
Case 1 ( $2^{\text {nd }}$ clause of the definition of a $N I_{\langle\alpha, \beta\rangle}$-inference): $\begin{gathered}\eta^{\prime} \\ A^{\prime}\end{gathered}$, where $\eta^{\prime}$ is an inference from a set of premises $\Gamma^{\prime}$ and $\Gamma$ is $\{A\} \cup \Gamma^{\prime}$.

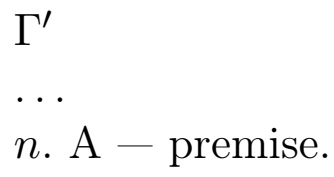

For $h\left(\eta^{\prime}\right)<h(\eta),{ }^{10}$ one can, by the inductive hypothesis, build up a $H I_{\langle\alpha, \beta\rangle}$-inference from a set of premises $\Gamma^{\prime}$. Then a $H I_{\langle\alpha, \beta\rangle}$-inference of $A$ from a set of premises $\Gamma$ looks as follows:

$\Gamma^{\prime}$

$\cdots$

$n^{\prime} . A-$ premise.

Case 2 ( $3^{r d}$ clause of the definition of a $N I_{\langle\alpha, \beta\rangle}$-inference): $\eta_{A^{\prime}}^{\prime}$, where $\eta^{\prime}$ is an inference of $C$ from a set of premises $\Gamma^{\prime}, \eta^{\prime}$ contains $A_{1}, \ldots, A_{k} ; A$ is inferred from $A_{1}, \ldots, A_{k}$ via one of the rules $\&_{e l 1}, \&_{e l 2}, \&_{i n}, \vee_{i n 1}, \vee_{i n 2}, \supset_{e l}$, and $\neg_{i n 1(\alpha)}$.

Subcase 2.1.: $\eta^{\prime}$ contains $\neg D$ and $D ; A$ is inferred from $\neg D$ and $D$ via $\neg_{\text {in } 1(\alpha)}$, where $j<n-1$ and $m<n-1$.

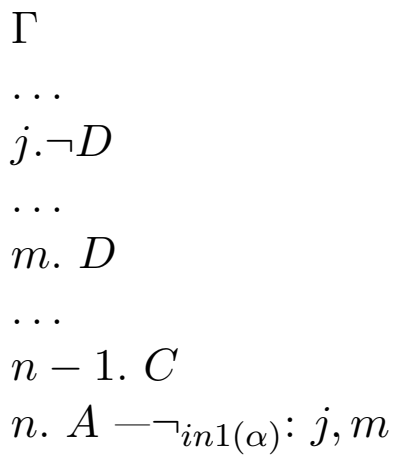

Let $\eta^{\prime}$ be an $N I_{\langle\alpha, \beta\rangle}$-inference of $C$ from $\Gamma, \eta_{1}^{\prime}$ be an $N I_{\langle\alpha, \beta\rangle}$-inference of $D$ from $\Gamma$, and $\eta_{2}^{\prime}$ be an $N I_{\langle\alpha, \beta\rangle}$-inference of $D$ from $\Gamma$, where $h\left(\eta_{1}^{\prime}\right)<h\left(\eta^{\prime}\right)$ and $h\left(\eta_{2}^{\prime}\right)<h\left(\eta^{\prime}\right)$, by the definition. The fact that $\mathrm{h}\left(\eta^{\prime}\right)<\mathrm{h}(\eta)$, implies that $h\left(\eta_{1}^{\prime}\right)<h(\eta)$ and $h\left(\eta_{2}^{\prime}\right)<(\eta)$, and, by the inductive hypothesis, one can build up the following $H I_{\langle\alpha, \beta\rangle}$-inferences:

\footnotetext{
${ }^{10}$ By the definition, $h\left(\begin{array}{c}\eta_{A}^{\prime} \\ A\end{array}\right)=h\left(\eta^{\prime}\right)+1$.
} 
a $H I_{\langle\alpha, \beta\rangle}$-inference of $D$ from $\Gamma$, a $H I_{\langle\alpha, \beta\rangle}$-inference of $D$ from $\Gamma$, and a $H I_{\langle\alpha, \beta\rangle}$-inference of $C$ from $\Gamma$. Then a $H I_{\langle\alpha, \beta\rangle}$-inference of $\mathrm{A}$ from a set of premises $\Gamma$ looks as follows:

$$
\begin{aligned}
& \Gamma \\
& \cdots \\
& j^{\prime} \cdot \neg D \\
& \cdots \\
& m^{\prime} . D \\
& \cdots \\
& n^{\prime}-1 . C \\
& n^{\prime} \cdot \neg D \supset(D \supset A)-H I_{\langle\alpha, \beta\rangle} \text {-axiom }(\mathrm{XI}, \alpha) \\
& \mathrm{n}^{\prime}+1 . A-\operatorname{modus} \text { ponens: } j^{\prime}, m^{\prime}, n^{\prime} \text { (two times) }
\end{aligned}
$$

Subcase 2.2.: $\eta^{\prime}$ contains $A \& B ; A$ is inferred from $A \& B$ via $\&_{e l 1}$, where $m<n-1$.

$$
\begin{aligned}
& \Gamma \\
& \ldots \\
& m . A \& B \\
& \ldots \\
& n-1 . C \\
& n . A-\&_{e l 1}: m
\end{aligned}
$$

Let $\eta^{\prime}$ be an $N I_{\langle\alpha, \beta\rangle}$-inference of $C$ from $\Gamma$ and $\eta_{1}^{\prime}$ be an $N I_{\langle\alpha, \beta\rangle}$-inference of $A \& B$ from $\Gamma$, where $h\left(\eta_{1}^{\prime}\right)<h\left(\eta^{\prime}\right)$, by the definition. The fact that $h\left(\eta^{\prime}\right)<h(\eta)$, implies that $h\left(\eta_{1}^{\prime}\right)<h(\eta)$ and, by the inductive hypothesis, one can build up the following $H I_{\langle\alpha, \beta\rangle}$-inferences: a $H I_{\langle\alpha, \beta\rangle}$-inference of $A \& B$ from $\Gamma$, a $H I_{\langle\alpha, \beta\rangle}$-inference of $C$ from $\Gamma$. Then a $H I_{\langle\alpha, \beta\rangle}$-inference of $A$ from a set of premises $\Gamma$ looks as follows:

$$
\begin{aligned}
& \Gamma \\
& \cdots \\
& m^{\prime} . A \& B \\
& \cdots \\
& n^{\prime}-1 . C \\
& n^{\prime} .(A \& B) \supset A-\mathrm{HI}_{\langle\alpha, \beta\rangle} \text {-axiom }(\mathrm{V})
\end{aligned}
$$




$$
n^{\prime}+1 . A-\operatorname{modus} \text { ponens: } m^{\prime}, n^{\prime}
$$

Subcase 2.3., where $\eta^{\prime}$ contains $B \& A ; A$ is inferred from $B \& A$ via $\&_{e l 2}$, is treated analogously to subcase 2.2 .

Subcase 2.4.: $\eta^{\prime}$ contains $B$ and $D ; A$ is $B \& D$ and is inferred from $B$ and $D$ via $\&_{i n}$, where $f<m, j<n-1$, and $m<n-1$.

$$
\begin{aligned}
& \Gamma^{\prime} \\
& \ldots \\
& j . B \\
& \ldots \\
& m . D \\
& \ldots \\
& n-1 . C \\
& \text { n. } B \& D-\&_{i n}: j, m
\end{aligned}
$$

Let $\eta^{\prime}$ be an $N I_{\langle\alpha, \beta\rangle}$-inference of $C$ from $\Gamma, \eta^{\prime} 1$ be an $N I_{\langle\alpha, \beta\rangle}$-inference of $B$ from $\Gamma$, and $\eta^{\prime} 2$ be an $N I_{\langle\alpha, \beta\rangle}$-inference of $D$ from $\Gamma$, where $h\left(\eta_{1}^{\prime}\right)<h\left(\eta^{\prime}\right)$ and $h\left(\eta_{2}^{\prime}\right)<h\left(\eta^{\prime}\right)$, by the definition. The fact that $h\left(\eta^{\prime}\right)<h(\eta)$, implies that $h\left(\eta_{1}^{\prime}\right)<h(\eta)$ and $h\left(\eta_{2}^{\prime}\right)<h(\eta)$, and, by the inductive hypothesis, one can build up the following $H I_{\langle\alpha, \beta\rangle}$-inferences: a $H I_{\langle\alpha, \beta\rangle}$-inference of $B$ from $\Gamma$, a $H I_{\langle\alpha, \beta\rangle}$-inference of $D$ from $\Gamma$, and a $H I_{\langle\alpha, \beta\rangle}$-inference of $C$ from $\Gamma$. Then a $H I_{\langle\alpha, \beta\rangle}$-inference of $B \& D$ from $\Gamma$ looks as follows:

$$
\begin{aligned}
& \Gamma \\
& \ldots \\
& f^{\prime} . A_{1}-\text { any } H I_{\langle\alpha, \beta\rangle} \text {-axiom } \\
& f^{\prime}+1 . B \supset\left(A_{1} \supset B\right)-H I_{\langle\alpha, \beta\rangle} \text {-theorem } \\
& f^{\prime}+2 . D \supset\left(A_{1} \supset D\right)-H I_{\langle\alpha, \beta\rangle} \text {-theorem } \\
& \ldots \\
& j^{\prime} . B \\
& j^{\prime}+1 . A_{1} \supset B-\text { modus ponens: } f^{\prime}+1, j^{\prime} \\
& \ldots \\
& m^{\prime} . D \\
& m^{\prime}+1 . A_{1} \supset D-\text { modus ponens: } f^{\prime}+2, m^{\prime}
\end{aligned}
$$




$$
\begin{aligned}
& n^{\prime}-1 . C \\
& n^{\prime} .\left(A_{1} \supset B\right) \supset\left(\left(A_{1} \supset D\right) \supset\left(A_{1} \supset(B \& D)\right)\right)-H I_{\langle\alpha, \beta\rangle} \text {-axiom }
\end{aligned}
$$

$$
n^{\prime}+1 . B \& D-\text { modus ponens: } j+1^{\prime}, m+1^{\prime}, f^{\prime}, n^{\prime} \text { (three times) }
$$

Subcase 2.5.: $\eta^{\prime}$ contains $B ; A$ is $B \vee D$ and is inferred from $B$ via $\vee_{i n 1}$, where $\mathrm{m}<n-1$.

$$
\begin{aligned}
& \Gamma \\
& \ldots \\
& \text { m. B } \\
& \ldots \\
& n-1 . C \\
& \text { n. } B \vee D-\vee_{i n 1}: m
\end{aligned}
$$

Let $\eta^{\prime}$ be an $N I_{\langle\alpha, \beta\rangle}$-inference of $C$ from $\Gamma$ and $\eta_{1}^{\prime}$ be an $N I_{\langle\alpha, \beta\rangle}$-inference of $B$ from $\Gamma$, where $h\left(\eta_{1}^{\prime}\right)<h\left(\eta^{\prime}\right)$, by the definition. The fact that $h\left(\eta^{\prime}\right)<$ $h(\eta)$, implies that $h\left(\eta_{1}^{\prime}\right)<h(\eta)$ and, by the inductive hypothesis, one can build up the following $H I_{\langle\alpha, \beta\rangle}$-inferences: a $H I_{\langle\alpha, \beta\rangle}$-inference of $B$ from $\Gamma$, a $H I_{\langle\alpha, \beta\rangle}$-inference of $C$ from $\Gamma$. Then a $H I_{\langle\alpha, \beta\rangle}$-inference of $B \vee D$ from a set of premises $\Gamma$ looks as follows:

$$
\begin{aligned}
& \Gamma \\
& \cdots \\
& m^{\prime} . B \\
& \cdots \\
& n^{\prime}-1 . C \\
& n^{\prime} . B \supset(B \vee D)-H I_{\langle\alpha, \beta\rangle} \text {-axiom (II) } \\
& n^{\prime}+1 . B \vee D-\operatorname{modus} \text { ponens: } m^{\prime}, n^{\prime}
\end{aligned}
$$

Subcase 2.6., where $\eta^{\prime}$ contains $D ; A$ is $B \vee D$ and is inferred from $D$ via $\vee_{i n 2}$, is treated analogously to subcase 2.5 .

Subcase 2.7.: $\eta^{\prime}$ contains $B \supset A$ and $B ; A$ is inferred from $B \supset A$ and $B$ via $\supset_{e l}$, where $j<n-1$, and $m<n-1$. 


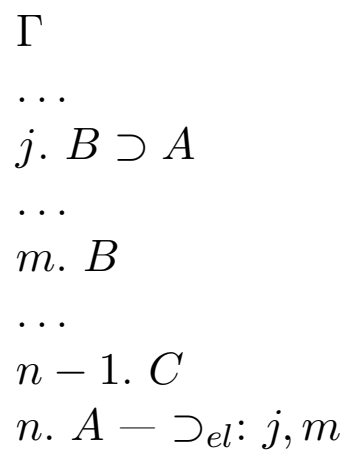

Let $\eta^{\prime}$ be an $N I_{\langle\alpha, \beta\rangle}$-inference of $C$ from $\Gamma, \eta_{1}^{\prime}$ be an $N I_{\langle\alpha, \beta\rangle}$-inference of $B \supset A$ from $\Gamma$, and $\eta_{2}^{\prime}$ be an $N I_{\langle\alpha, \beta\rangle}$-inference of $B$ from $\Gamma$, where $h\left(\eta_{1}^{\prime}\right)<h\left(\eta^{\prime}\right)$ and $h\left(\eta_{2}^{\prime}\right)<h\left(\eta^{\prime}\right)$, by the definition. The fact that $h\left(\eta^{\prime}\right)<$ $h(\eta)$, implies that $h\left(\eta_{1}^{\prime}\right)<h(\eta)$ and $h\left(\eta_{2}^{\prime}\right)<h(\eta)$, and, by the inductive hypothesis, one can build up the following $H I_{\langle\alpha, \beta\rangle}$-inferences: a $H I_{\langle\alpha, \beta\rangle^{-}}$ inference of $B \supset A$ from $\Gamma$, a $H I_{\langle\alpha, \beta\rangle}$-inference of $B$ from $\Gamma$, and a $H I_{\langle\alpha, \beta\rangle}$-inference of $C$ from $\Gamma$. Then a $H I_{\langle\alpha, \beta\rangle}$-inference of $A$ from $\Gamma$ looks as follows:

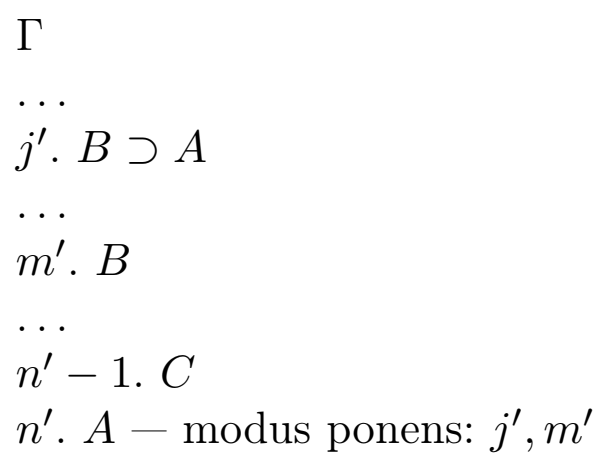

Case 3 ( $4^{\text {th }}$ clause of the definition of a $N I_{\langle\alpha, \beta\rangle}$-inference). $A$ is $B \supset C$ and a $N I_{\langle\alpha, \beta\rangle}$-inference $\eta$ of a height $n$ of $B \supset C$ from $\Gamma$ looks as follows: $\underset{B \supset C}{\mid \gamma}$, where $\gamma_{1}$ is a $N I_{\langle\alpha, \beta\rangle}$-inference $\iota$ of $C$ from $\{B\} \cup \Gamma$, $\gamma_{1}$ is a part of $\iota$, starting from the last premise $B$ in $\iota$ until $C$, itself, and $m<n-1$.

$\Gamma$

..

|m. $B-$ premise 


$$
\begin{aligned}
& \mid \ldots \\
& \mid n-1 . C \\
& \text { n. } B \supset C-\supset_{i n}: n-1
\end{aligned}
$$

For $h(\iota)<h(\eta)$, one can, by the inductive hypothesis, ${ }^{11}$ build up a $H I_{\langle\alpha, \beta\rangle}$-inference of $C$ from $\{B\} \supset \Gamma$. Then a $H I_{\langle\alpha, \beta\rangle}$-inference of $B \supset C$ from $\Gamma$ looks as follows:

$$
\begin{aligned}
& \Gamma \\
& \cdots \\
& m^{\prime} . B \text { - premise } \\
& \cdots \\
& n^{\prime}-1 . C \\
& n^{\prime} . B \supset C-\text { deduction theorem: } m^{\prime}, n^{\prime}-1
\end{aligned}
$$

Case $4\left(5^{\text {th }}\right.$ clause of the definition of a $N I_{\langle\alpha, \beta\rangle}$-inference). A $N I_{\langle\alpha, \beta\rangle}$-inference $\eta$ of a height $n$ of $A$ from $\Gamma$ looks as follows: ${ }_{A}^{\gamma} \gamma_{1}$, where $\underset{\gamma_{1}}{\gamma}$ is a $N I_{\langle\alpha, \beta\rangle}$-inference $\iota$ of $A$ from $\{A \supset B\} \cup \Gamma, \gamma_{1}$ is a part of $\iota$, starting from the last premise $A \supset B$ in $\iota$ until $A$, itself, and $\mathrm{m}<n-1$.

$$
\begin{aligned}
& \Gamma \\
& \text { ‥ } \\
& \mid \ldots . A \supset B-\text { premise } \\
& \mid \ldots \\
& \mid n-1 . A \\
& n . A-\supset_{P}: n-1
\end{aligned}
$$

For $h(\iota)<h(\eta)$, one can, by the inductive hypothesis, build up a $H I_{\langle\alpha, \beta\rangle^{-}}$ inference of $A$ from $\{A \supset B\} \cup \Gamma$. Then a $H I_{\langle\alpha, \beta\rangle}$-inference of $A$ from $\Gamma$ looks as follows:

$$
\begin{aligned}
& \Gamma \\
& \cdots \\
& m^{\prime} . A \supset B-\text { premise }
\end{aligned}
$$

\footnotetext{
${ }^{11}$ Here and in the cases below, we stress the fact that we proceed from one inference to another inference, not from a part of an inference to another inference. So, the inductive hypothesis of the theorem is applicable.
} 


$$
\begin{aligned}
& \cdots \\
& n^{\prime}-1 . A \\
& n^{\prime} .(A \supset B) \supset A-\text { deduction theorem: } m^{\prime}, n^{\prime}-1 \\
& n^{\prime}+1 .((A \supset B) \supset A) \supset A-H I_{\langle\alpha, \beta\rangle} \text {-axiom }(\mathrm{X}) \\
& n^{\prime}+2 . A-\text { modus ponens: } n^{\prime}, n^{\prime}+1
\end{aligned}
$$

Case 5 ( $6^{\text {th }}$ clause of the definition of a $N I_{\langle\alpha, \beta\rangle}$-inference). $A$ is $\neg E$, where $E$ is a formula which is not a quasi-elemental formula of a length less than $\beta$, and a $N I_{\langle\alpha, \beta\rangle}$-inference $\eta$ of a height $n$ of $\neg E$ from $\Gamma$ looks as follows: $\underset{\neg E}{\mid \gamma}$, where $\underset{\gamma}{\gamma}$ is a $N I_{\langle\alpha, \beta\rangle}$-inference $\iota$ of $\neg(A \supset A)$ from $\{E\} \cup \Gamma$, $\gamma_{1}$ is a part of $\iota$, starting from the last premise $E$ in $\iota$ until $\neg(A \supset A)$, itself, and $m<n-1$.

$$
\begin{aligned}
& \Gamma \\
& \ldots \\
& \mid m . E-\text { premise } \\
& \mid \ldots \\
& \mid n-1 . \neg(A \supset A) \\
& n . \neg E-\neg \operatorname{in} 2(\beta): n-1
\end{aligned}
$$

For $h(\iota)<h(\eta)$, one can, by the inductive hypothesis, build up a $H I_{\langle\alpha, \beta\rangle^{-}}$ inference of $\neg(A \supset A)$ from $\{E\} \cup \Gamma$. Then a $H I_{\langle\alpha, \beta\rangle}$-inference of $A$ from $\Gamma$ looks as follows:

$$
\begin{aligned}
& \Gamma \\
& \cdots \\
& m^{\prime} . E-\text { premise } \\
& \cdots \\
& n^{\prime}-1 . \neg(A \supset A) \\
& n^{\prime} . E \supset \neg(A \supset A)-\text { deduction theorem: } m^{\prime}, n^{\prime}-1 \\
& n^{\prime}+1 .(E \supset \neg(A \supset A)) \supset \neg E-H I_{\langle\alpha, \beta\rangle} \text {-axiom }(\mathrm{XII}, \beta) \\
& n^{\prime}+2 . A-\operatorname{modus} \text { ponens: } n^{\prime}, n^{\prime}+1
\end{aligned}
$$

Case 6 ( $7^{\text {th }}$ clause of the definition of a $N I_{\langle\alpha, \beta\rangle}$-inference). a $N I_{\langle\alpha, \beta\rangle^{-}}$ inference $\eta$ of a height $n$ of $A$ from $\Gamma$ looks as follows: $\begin{gathered}\gamma \\ \mid \gamma_{1}\end{gathered}$, where $\underset{A}{\gamma_{2}} \underset{\gamma_{2}}{\gamma}$ is a $N I_{\langle\alpha, \beta\rangle}$-inference $\iota$ of A from $\{D\} \cup\{\mathrm{B}\} \cup \Gamma, \gamma$ contains $D \vee \mathrm{B}, \gamma_{1}$ is a part 
of $\eta$, starting from a premise $D$ in $\eta$ until $\mathrm{A}, \gamma_{2}$ is a part of $\eta$, starting from the last premise $\mathrm{B}$ in $\eta$ until $A$, itself, and $f<g, g<j, j<n-1$. $^{12}$

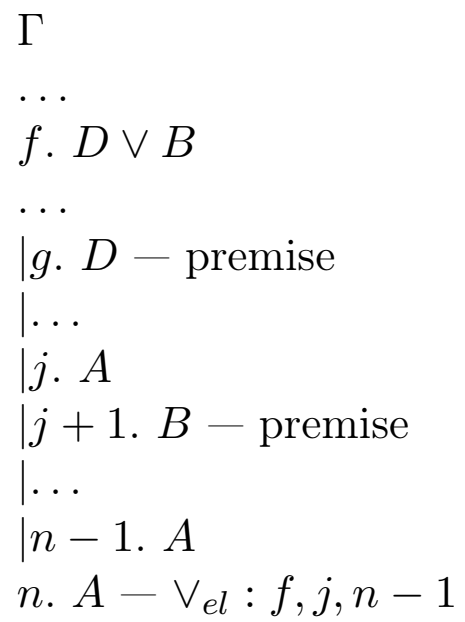

First, let us consider a $N I_{\langle\alpha, \beta\rangle}$-inference $\iota$ of $A$ from $\{D\} \cup\{\mathrm{B}\} \cup \Gamma$.

$\Gamma$

$\cdots$

f. $D \vee B$

...

g. $D$ - premise

$\cdots$

j. A

$j+1 . B-$ premise

$n-1 . A$

By the construction, $\iota$ contains the following $N I_{\langle\alpha, \beta\rangle}$-inferences: $\iota_{1}$ of $D \vee B$ from $\Gamma, \iota_{2}$ of $A$ from $\{D\} \cup \Gamma$, and $\iota_{3}$ of $A$ from $\{B\} \cup\{D\} \cup \Gamma$. For $h\left(\iota_{i}\right)<h(\iota),{ }^{13}$ for each $i$ from $\{1,2,3\}$, one can build up a $H I_{\langle\alpha, \beta\rangle}$-inference of $D \vee B$ from $\Gamma$, a $H I_{\langle\alpha, \beta\rangle}$-inference of A from $\{D\} \cup \Gamma$,

\footnotetext{
${ }^{12}$ On alternatives of this case see the footnote to the $7^{\text {th }}$ clause of the definition of an $N I_{\langle\alpha, \beta\rangle}$-inference.

${ }^{13}$ Unlike the other cases, this case requires the inductive hypothesis holds true for a $N I_{\langle\alpha, \beta\rangle}$-inference of any length less than the length of $\iota$, not only for a $N I_{\langle\alpha, \beta\rangle}$ inference of a length $h(\iota)-1$.
} 
and a $H I_{\langle\alpha, \beta\rangle}$-inference of $\mathrm{A}$ from $\{B\} \cup\{D\} \cup \Gamma$. Then a $H I_{\langle\alpha, \beta\rangle}$-inference of $\mathrm{A}$ from $\{D\} \cup\{\mathrm{B}\} \cup \Gamma$ looks as follows:

$\Gamma$

$\cdots$

$f^{\prime} . D \vee B$

...

$g^{\prime} . D-$ premise

$\cdots$

$j^{\prime} . A$

$j^{\prime}+1 . B-$ premise

$n^{\prime}-1 . A$

So, a $H I_{\langle\alpha, \beta\rangle}$-inference of $\mathrm{A}$ from $\Gamma$ looks as follows:

$\Gamma$

...

$f^{\prime} . D \vee B$

‥

$g^{\prime} . D-$ premise

$j^{\prime} . A$

$j^{\prime}+1 . B-$ premise

$n^{\prime}-1 . A$

$n^{\prime} . B \supset A-$ deduction theorem: $j^{\prime}+1, n^{\prime}-1$

$\Gamma$

$f^{\prime \prime} . D \vee B$

...

$g^{\prime \prime} . D$ - premise

$j^{\prime \prime} . A$

$j^{\prime \prime}+1 . D \supset A-$ deduction theorem: $g^{\prime \prime}, j^{\prime \prime}$

$j^{\prime \prime}+2 .(D \supset A) \supset((B \supset A) \supset((D \vee B) \supset A))-H I_{\langle\alpha, \beta\rangle^{-}}$ axiom (IV) 


$$
j^{\prime \prime}+3 . A-\supset_{e l}: f^{\prime \prime}, j^{\prime \prime}+1, j^{\prime \prime}+2 \text { (three times) }
$$

The Theorem implies a Corollary: for each $\alpha, \beta \in\{0,1,2,3, \ldots \omega\}$, a Hilbert-style calculus $H I_{\langle\alpha, \beta\rangle}$ and a ND system $N I_{\langle\alpha, \beta\rangle}$ are equipollent, i.e., $\mathrm{A}$ is a $H I_{\langle\alpha, \beta\rangle}$-theorem iff $\mathrm{A}$ is a $N I_{\langle\alpha, \beta\rangle}$-theorem.

\section{Final remarks}

In the paper, for each logic, $I_{\langle\alpha, \beta\rangle}, \alpha, \beta \in\{0,1,2,3, \ldots \omega\}$, such that $I_{\langle 0,0\rangle}$ is propositional classical logic [10], we, continuing the series of works $[8,9]$, present a subordinated ND system $N I_{\langle\alpha, \beta\rangle}$. Moreover, each ND system has a precise definition of an inference which is a modification of V. Smirnov's approach. Our approach highlights a view on the implication introduction rule as the genus for the other indirect rules. Using a Hilbert-style calculus $H I_{\langle\alpha, \beta\rangle}$, for each logic in question, presented by V. Popov [10], we show that a formula A is provable in $H I_{\langle\alpha, \beta\rangle}$ iff it is provable in $N I_{\langle\alpha, \beta\rangle}$. In the future, we point out studying consequences of the precise definition with an application to complexity problems [7]. Last, not least, we look forward to formulating proof searching procedures for these ND systems in the fashion of $[3,4]$.

\section{Corrections}

The paper "Natural deduction in a paracomplete setting" by A. Bolotov and V. Shangin to have been published in this Journal's $20^{t h}$ volume needs two corrections. First, the $23^{r d}$ entry in the references list should be replaced with "Popov V. and Solotschenkov A. Semantics of propositional paracomplete Nelson logic // Integrated scientific journal. V. 8. 2012. P. 31-32. (In Russian)". Second, the truth-table definitions for the connectives of logic PComp in the $2^{\text {nd }}$ section must be added with the following footnote: A. Avron had told V. Popov about these definitions at the World Congress on Paraconsistency (Ghent, 1997) and then V. Popov told one of the paper's authors about these definitions.

\section{Acknowledgments}

The author thanks the referees for commenting the previous draft of the paper. 


\section{References}

[1] Anisov, A.M. Modern logic. Moscow: IFRAN Publishers, 2002. 273 pp. (In Russian)

[2] Bocharov, V.A., Markin, V.I. Introduction to logic. Moscow: Forum Publ. house INFRA-M Publ., 2011. 560 pp. (In Russian)

[3] Bolotov, A., Shangin, V. "Natural Deduction System in Paraconsistent Setting: Proof Search for PCont", Journal of Intelligent Systems, 2012, Vol. 21(1), pp. 1-24.

[4] Bolotov, A., Basukoski, A., Grigoriev, O., Shangin, V. "Natural deduction calculus for linear-time temporal logic", LNAI, 2006, Vol. 4160, pp. 56-68.

[5] Gentzen, G. The collected papers, ed. M.E. Szabo. North-Holland Pub. Co., 1969. 338 pp.

[6] Ivlev, Yu.V. Logic. Moscow: Prospect Publ., 2008. 304 pp. (In Russian)

[7] Kozhemyachenko, D. "Simulation of natural and sequent calculi", Logicphilosophical studies, 2016, Vol. 13(2), pp. 181-182. (in Russian)

[8] Popov, V., Shangin, V. "Syntax and semantics of simple paracomplete logics", Logical investigations, 2013, Vol. 19, pp. 325-334.

[9] Popov, V., Shangin, V. "Syntax and semantics of simple paranormal logics", Logical-philosophical studies, 2014, Vol. 6, pp. 290-297.

[10] Popov, V. "On one generalization of Glivenko theorem", Logical investigations, 2015, Vol. 21(1), pp. 100-121. (In Russian)

[11] Quine, W.V. "On natural deduction", The Journal of Symbolic Logic, 1950, Vol. 15, No. 2, pp. 93-102.

[12] Smirnov, V.A. Formal inference and logical calculi. Moscow: Nauka Publishers, 1972. (In Russian)

[13] Smirnov, V.A., Anisov, A.M., Arutyunov, G.P., Dmitriyev, D.V., Melentyev, A.S., and Mikhailov, F.T. Logic and clinical diagnostics. Theoretical foundations. Moscow: Nauka Publishers, 1994. 271 pp. (In Russian)

[14] Smirnov, V.A., Markin, V.I., Novodvorsky, A.E., and Smirnov, A.V. "Proof and proof searching", in: Logic and computer, Vol. 3. Moscow: Nauka Publ., 1996. 296 pp. (In Russian)

[15] Tomova, N.E., Shalack, V.I. Introduction to logic for philosophy faculties' students. Moscow: IFRAN Publishers, 2014. 191 pp. (In Russian)

[16] Voishvillo, E.K. Concept as a form of thinking. Moscow: MSU Publishers, 1989. 239 pp. (In Russian) 